\title{
On the structure of periodic complex Horadam orbits
}

\section{Ovidiu D. Bagdasar, Peter J. Larcombe and Ashiq Anjum}

\begin{abstract}
.
Numerous geometric patterns identified in nature, art or science can be generated from recurrent sequences, such as for example certain fractals or Fermat's spiral. Fibonacci numbers in particular have been used to design search techniques, pseudo random-number generators and data structures. Complex Horadam sequences are a natural extension of Fibonacci sequence to complex numbers, involving four parameters (two initial values and two in the defining recursion), therefore successive sequence terms can be visualized in the complex plane. Here, a classification of the periodic orbits is proposed, based on divisibility relations between orders of generators (roots of the characteristic polynomial). Regular star polygons, bipartite graphs and multi-symmetric patterns can be recovered for selected parameter values. Some applications are also suggested.
\end{abstract}

\section{REFERENCES}

[1] Albertson, M. O. and Hutchinson, J. P., Discrete Mathematics with Algorithms, John Wiley \& Sons, New York, 1988

[2] Bagdasar, O. and Larcombe, P. J., On the characterization of periodic complex Horadam sequences, Fibonacci Quart., 51 (2013), No. 1, 28-37

[3] Bagdasar, O. and Larcombe, P. J., On the number of complex periodic complex Horadam sequences, Fibonacci Quart., 51 (2013), No. 4, 339-347

[4] Bagdasar, O., Larcombe, P. J. and Anjum, A., Particular Orbits of Periodic Horadam Sequences, Octogon Math. Mag., 21, (2013) No. 1, 87-98

[5] Bagdasar, O. and Chen, M., A Horadam-based Pseudo-random Number Generator, Proceedings of 16th UKSim, Cambridge (2014), 226-230

[6] Bagdasar, O. and Larcombe, P. J., On the characterization of periodic generalized Horadam sequences, J. Differ. Equ. Appl., 20 (2014), No. 7, 1069-1090

[7] Cassandras, C. G. and Lafortune, S., Introduction to Discrete Event Systems, Springer (2nd Ed.), 2007

[8] Coxeter, H. S. M., Introduction to Geometry, New York Wiley (2nd Ed.), 1969

[9] Fredman, M. L. and Tarjan, R. E. Fibonacci heaps and their uses in improved network optimization algorithms, Journal Assoc. Comput. Mach., 34 (1987), No. 3, 596-615

[10] Horadam, A. F., Basic properties of a certain generalized sequence of numbers, Fibonacci Quart., 3 (1965), 161-176

[11] Koshy, T., Fibonacci and Lucas Numbers with Applications, John Wiley \& Sons, Inc., Hoboken, NJ, USA, 2001

[12] Knuth, D. E., The Art of Computer Programming, Addison Wesley (2nd Ed.), 3/2003

[13] Larcombe, P. J., Bagdasar, O. D. and Fennessey, E. J., Horadam sequences: a survey, Bull. Inst. Combin. Appl, 67 (2013), 49-72

[14] Latapy, M., Phan, T. H. D., Crespelle, C. and Nguyen, T. Q., Termination of Multipartite Graph Series Arising from Complex Network Modelling, in Combinatorial Optimization and Applications, Lecture Notes in Computer Science, 6508 (2010), 1-10

[15] Noonea, C. J., Torrilhonb, M. and Mitsosa, A., Heliostat field optimization: A new computationally efficient model and biomimetic layout, Solar Energy, 86 (2012), No. 2, 792-803

[16] The On-Line Encyclopedia of Integer Sequences, http:/ / oeis.org, OEIS Foundation Inc. (2011)

Received: 10.08.2014; In revised form: 19.11.2014; Accepted: 26.11.2014

2010 Mathematics Subject Classification. 11B37, 11B39, 51M15.

Key words and phrases. Linear recurrence, complex Horadam sequence, periodicity, geometric patterns.

Corresponding author: Ovidiu Bagdasar; o.bagdasar@derby.ac.uk 
DEPARTMENT OF COMPUTING AND MATHEMATICS

UNIVERSITY OF DERBY

Kedleston RoAd, DE22 1GB, Derby, United Kingdom

E-mail address: $\circ$.bagdasar@derby . ac.uk

E-mail address: p.j. larcombe@derby . ac.uk

E-mail address: a. an jum@derby . ac.uk 